\title{
EAl Endorsed Transactions

\section{Individual recommendation method of college physical education resources based on cognitive diagnosis model}

\author{
Hongming Guo ${ }^{1}$ and Xiaochun Cheng ${ }^{2, *}$ \\ ${ }^{1}$ Department of Sports, Wuxi Vocational Institute of Commerce, Wuxi Jiangsu 214000, China; guohongming@ wxic.edu.cn \\ ${ }^{2}$ Department of Computer Science, Middlesex University, London NW4 4BT, United Kingdom; x.cheng@ @mdx.ac.uk
}

\begin{abstract}
In order to improve the safety of college physical education resources recommendation and reduce the test overlap rate and resource exposure rate, a personalized recommendation method of college P.E. teaching resources based on cognitive diagnosis model is proposed. A cognitive diagnosis model based on multi-level attribute score is designed to model students' resource mastery level according to existing answers and the relevance of knowledge points. The knowledge mastery attribute model of the tested students is used for probability matrix decomposition to predict the students' answers, and make corresponding resource recommendations according to the score prediction and resource difficulty. Experiments show that resource exposure value of the method in this paper is lower than 1 , and its security is high. Regarding the experiment of overlapping indicators, the value of the test overlap rate of the method in this paper is always lower than 0.01 , and the recommended resources are more accurate. The F1 value of the method in this paper is up to 0.98 , and the deviation of resource recommendation is small. And the real-time performance is high after the method is applied, and the phenomenon of cold start will not occur.
\end{abstract}

Keywords: Cognitive diagnosis model, College physical education, Teaching resources, Personalized recommendation, Probability matrix decomposition, Knowledge mastery attribute.

Received on 11 December 2021, accepted on 18 January 2022, published on 10 February 2022

Copyright (C) 2022 Hongming Guo et al., licensed to EAI. This is an open access article distributed under the terms of the Creative Commons Attribution license, which permits unlimited use, distribution and reproduction in any medium so long as the original work is properly cited.

doi: 10.4108/eai.10-2-2022.173379

*Corresponding author. x.cheng@mdx.ac.uk

\section{Introduction}

Research on the Teaching Reform Path of Practical Courses in Higher Vocational Colleges under the ActionOriented Teaching Mode Nowadays, some colleges and universities have already applied multimedia teaching systems to teaching, and the use of multimedia teaching systems has been very common a long time ago. Students learn through the teaching system, they can also communicate with teachers, and we can share teaching resources together. The core of the learning method is the use of multimedia interactive teaching, and the guidance is employment, so that students can consciously devote themselves to learning. However, most resource search engines only rely on keyword retrieval, and the effect of resource recommendation is not ideal [1]. Cognitive diagnosis can identify students' current cognitive state and make up for the shortcomings of classical measurement theory and item response theory. It can be said that cognitive diagnosis opens the "black box" of students' cognitive structure [2]. At present, there are more than 70 kinds of cognitive diagnosis models developed at home and abroad, including linear logic sticker trait model (LLTM), multi-component latent trait model (MLTM), multidimensional item response theory (MIRT), the deterministic (Unified), the noise, or gate model, the Bayesian network (Bayesian Network), the unified model, and the fusion model, etc. [3].

Modern physical education (P.E.) online education is very different from traditional P.E. classroom education. Their difference lies in whether to conduct targeted mining and analysis of students' historical learning, obtain students' personalized learning characteristics, and 
achieve the purpose of personalized recommendation of P.E. teaching resources [4]. Personalized learning features include learning objectives set by students for learning needs, learning styles and habits formed in specific learning stages, cognitive state and ability level [5]. In the actual P.E. teaching resources recommendation, according to these differences in personality characteristics, targeted teaching resources recommendation strategies are adopted to optimize the learning path, so as to realize personalized teaching services. In addition, personalized learning also plays an important role in establishing the relationship between learners' existing cognition and unknown learning state [6]. According to the unique individual differences of students, it can guide and help students find their own advantages and disadvantages, so as to realize the all-round development of students in the teaching process.

The core of completing and realizing personalized learning is to comprehensively consider the recommendation of physical education teaching resources such as different students' situational factors and their own psychological cognitive factors [7]. Its purpose is to fully tap learners' personality and optimize learners' learning process while following the development law of P.E. teaching field and taking personalized learning theory as the guiding direction, so as to improve the process of learning efficiency. Therefore, it is particularly important to pay real-time attention to and dynamically update students' cognitive level and what goals and needs students need to complete at each stage of the learning process. Many scholars have studied personalized recommendation methods, for example, Wei et al. [8] studied personalized information recommendation based on synonym label optimization, but in the recommendation method, the impact of data sparsity on the recommendation effect was not considered. Wu et al. [9] studied personalized recommendation based on the consumption psychology of local group purchase ecommerce users, but when new resources appeared, the lack of corresponding real-time update ability lead to inaccurate recommendation results. Zhou et al. [10] studied personalized recommendation through user preference matching, but this method would lose some students' own learning characteristics when predicting students' performance. Therefore, this paper studies the personalized recommendation method of college P.E. teaching resources based on cognitive diagnosis mode. We first construct a cognitive diagnostic model based on multi-level attribute scoring. This paper calculates the correlation between students' answers and resource knowledge points, so as to grasp the students' learning level. And the PMF-CD method is used to predict students' scores and complete the recommendation of personalized physical education resources.

\section{Personalized recommendation of college P.E. teaching resources}

\subsection{Overall structure of personalized recommendation based on cognitive diagnosis}

In this paper, the personalized recommendation method of college P.E. teaching resources based on cognitive diagnosis model includes three parts: collecting answer data of P.E. teaching resources, cognitive diagnosis and personalized recommendation. Firstly, according to the needs of students, collect the resources that students have answered in P.E. teaching resources. These answered resources constitute the data source of cognitive diagnosis, that is, cognitive diagnosis is based on the situation that these resources have been selected and not selected [11]. The next step is cognitive diagnosis. At present, there are many cognitive diagnosis models that can support cognitive diagnosis. Although the specific diagnosis methods of different cognitive diagnosis models are different, their core ideas are basically similar. Therefore, in the implementation of the recommended method, the basic process supporting cognitive diagnosis is the same [12]. The result of cognitive diagnosis is to output the current cognitive structure of students, and get which of all college P.E. teaching resources have been selected by students and which have not been selected by students. Personalized recommendation based on cognitive diagnosis is based on the results of cognitive diagnosis. Since cognitive diagnosis essentially uses statistical methods to classify students' current cognitive structure [13] and classify students into a certain type they may belong to, when making personalized recommendation, it only needs to formulate push strategies for each different cognitive structure type to realize personalized recommendation for each student. The personalized recommendation method framework designed in this paper is shown in Figure 1.

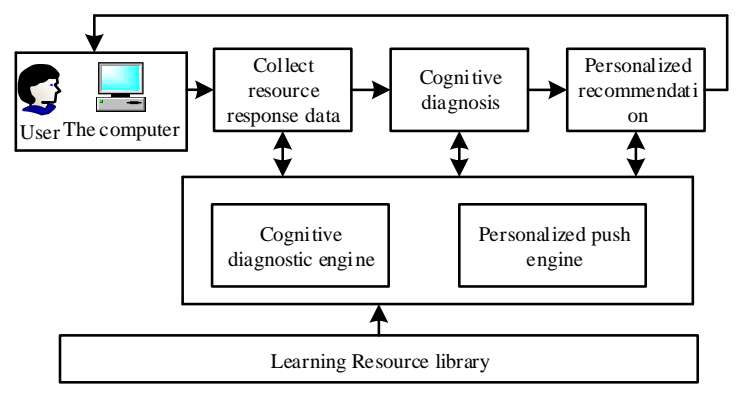

Figure 1. Personalized recommendation method framework 
The basic framework of personalized recommendation of college P.E. teaching resources based on cognitive diagnosis model is shown in Figure 1. The implementation of cognitive diagnosis and personalized recommendation requires the support of learning resource database, and the data scheduling and program execution under the management of cognitive diagnosis engine and personalized recommendation engine.

In the learning resource library, test question resources required for diagnosis and various types of learning resources for personalized recommendation are stored, including video resources, text resources, pictures, animation, etc. In order to realize cognitive diagnosis and personalized resource recommendation, we must establish a learning resource model to identify different learning resources, so as to realize the dynamic extraction and push of learning resources.

\subsection{Design of cognitive diagnosis model}

A cognitive diagnosis model is built based on the data in the learning resource database. In the process of practical application, people often need to evaluate the attribute level due to different cognitive structure and involved cognitive attribute components [14]. Therefore, in order to meet the actual complex application requirements and application environment and improve the accuracy and calculation efficiency of diagnosis and evaluation, this paper improves the P-DINA model. It is improved to a cognitive diagnosis model polytomous hierarchical DINA (PH-DINA) based on multi-level attribute score, which is used to model the response of students' resources. The improved cognitive diagnosis model can score according to multi-level attributes, and accurately recommend teaching resources according to learning resources and students' knowledge.

In order to facilitate the subsequent calculation of relevant models and frameworks, the expression of multilevel attribute teaching resources is defined. Since a single teaching resource has an objective phenomenon that multiple knowledge points can be assessed, that is, each resource contains at least one knowledge attribute [15], the test resource $q$ can be expressed as $q_{j}=\left(q_{j 1}, q_{j 2}, \ldots, q_{j o}\right)$, where $j$ is the number of topics in the test resource; $O$ is the maximum number of subject $j$ knowledge points to be assessed; $q_{j o}=\{0,1,2, \ldots, n\}$ means that the $o_{\text {-th test attribute }}$ has $n$ levels. If $q_{j o} \geq 1$, it means the ${ }^{o}$-th knowledge attribute with difficulty of $n$. On the contrary, $q_{j o}=0$ means that the knowledge attribute is not tested.

PH-DINA model
P-DINA is a typical non compensation model, that is, students participating in the test must master all skills or knowledge attributes $a_{i}$ to be tested in the resources before they can be considered to answer correctly. All skills or attributes tested by the project are included in the project $q_{j}$ to be tested. The response function of the project is:

$$
\begin{gathered}
P\left(Y_{i j}=t \mid a_{i}\right)=P *\left(Y_{i j}=t \mid a_{i}\right)-P *\left(Y_{i j}=t+1 \mid a_{i}\right) \\
P *\left(Y_{i j}=t \mid a_{i}\right)=\left(1-s_{j t}\right)^{\eta_{i j}} \cdot g_{j t}^{1-\eta_{i j}} \\
\eta_{i j}=\prod_{K=1}^{K} K \alpha_{i o}^{q_{j o}}
\end{gathered}
$$

In formula (2.1), $P\left(Y_{i j}=t \mid a_{i}\right)$ represents the probability that the tested student $i$ will score $t$ on item $j_{;} P^{*}\left(Y_{i j}=t \mid a_{i}\right)$ represents the probability that the student $i$ will score $t$ or above on item $j ; \eta_{i j} \in\{0,1\}$ represents the result score of the students when learning resources in an ideal state (regardless of mistakes); $s_{j}=P\left(Y_{i j}=1 \mid \eta_{i j}=1\right)$

$j$, which refers to the probability that the students still do the wrong questions in the item after mastering the attribute of item $j$ assessment; $g_{j}=P\left(Y_{i j}=1 \mid \eta_{i j}=0\right)$ is the guessing parameter of item $j$, which refers to the probability that the tested students complete the resource test questions when they have not fully mastered the attributes of the target $j$ assessment.

The guess parameters and error parameters in the above formula (2.2) need to meet the following requirements: $s_{j t} \leq s_{j t+1}$, that is, for the students who need to master the project $j$ 's assessment attribute, the error probability of getting $t$ score is less than that of getting $t+1$ score; $g_{j t} \leq g_{j t+1}$, that is, for the students who do not fully grasp the project $j$ assessment attributes, the probability of correctly guessing $t$ points is greater than that of correctly guessing $t+1$ points, so as to ensure that the probability of correctly answering is never negative.

In formula (2.3), $K$ represents the number of test attributes,

$$
\alpha_{i}=\left(\alpha_{i 1}, \alpha_{i 2}, \ldots, \alpha_{i o} \mid \alpha_{i o}=\{0,1\}\right)
$$


represents the mastery of each attribute by the tested student $i, \alpha_{i o}=1$ indicates that the tested student $i$ has mastered the attribute $O$, and vice versa is 0 , indicating that it has not mastered; $q_{j}=\left(q_{j 1}, q_{j 2}, \ldots, q_{j o} \mid q_{j o}=\{0,1\}\right)$ indicates that item $j$ has examined each attribute. $q_{j o}=1$ indicates that item $j$ has examined attribute $O$. Conversely, 0 indicates that item $j$ has not examined.

In order to apply to complex knowledge structure and practical multi-attribute resources, this paper introduces the concept of attribute hierarchy. The matrix $Q$ with multi-level attributes can be assigned any integer value. For example, $q_{j o}=3$ represents the attribute $o$ with item $j$ test index of 3 , and $\alpha_{i o}=2$ represents that the subject $i$ has mastered the second level of attribute $O$. If the tested students want to complete the project correctly, they need to master the assessment attribute index level and its above levels. For example, the attribute $p_{j}=(1,3,2)$ measured by project $j$ is 0 , and the attributes $A_{1}, A_{2}$ and $A_{3}$ have three levels of 2,4 and 3 respectively. Only when the tested students master the attribute

$a_{i}=\{(1,3,2)|(2,3,2)|(1,3,3)|(2,3,3)|(3,3,2) \mid(3,3,3)\}$

can they complete project $j$.

For the attribute multi-level model, there are $L_{o}$ values of $a_{i o}$ and $q_{j o}$, that is, there are $L \geq 2$ levels of attribute $O$. Therefore, if the value of attribute $O$ is a non-0-1 element, the ideal response score $\eta_{i j}$ and item response function are no longer applicable, and the difficulty and amount of calculation of parameter estimation are increased. In order to keep the simplicity and interpretability of cognitive diagnosis model, it is necessary to convert multi-level $\alpha$ and $q$ into 0-1 elements through Discriminant function. The discriminant function can accurately check whether the center of gravity of each learning resource group is different, and can effectively assist the multi-level processing of attributes. The Discriminant function is as follows:

$$
\alpha_{i o}^{\prime}=\left\{\begin{array}{l}
1, a_{i o} \geq q_{i o} \\
0, a_{i o}<q_{i o}
\end{array}, q^{\prime}{ }_{j o}=\left\{\begin{array}{l}
1, q_{j o} \geq 1 \\
0, q_{j o}=0
\end{array}\right.\right.
$$

At this time, although the model realizes the calculation and processing of attribute multi polarization and meets the objective examination requirements of multi-level attributes, the observation score still cannot correspond to the ideal score. Therefore, in order to further describe the real feedback of the subject students to master the familiar mode on the projec. Due to the imbalance of learning resource samples, the accuracy of resource recommendation will be affected. The Weight function can effectively adjust the weight of the sample and adjust the balance of the sample. Weight function is used to expand formula (3) into a multi-level ideal score function:

$$
\begin{gathered}
\eta_{i j}^{*}=\operatorname{round}\left(\sum_{o=1}^{K} \rho_{i j o} \cdot \omega_{j o} \cdot m f_{j}\right) \\
\rho_{i j o}=\left\{\begin{array}{l}
\frac{\alpha_{i o}^{\prime}}{q_{j o}^{\prime}}, q_{j o}^{\prime}=1 \\
0, q_{j o}^{\prime}=0
\end{array}\right.
\end{gathered}
$$

In the above formula, $\omega_{j o}$ is the weight of $O$ attribute in the test attribute of item $j, \frac{\alpha_{i o}^{\prime}}{q_{j o}^{\prime}}$ is the proportion of students $i$ mastering familiarity with item $j$, and $m f_{j}$ is the full score of item $j$. In order to facilitate parameter estimation and reduce the calculation scale of the model, formula (2.2) is transformed into:

$$
\begin{gathered}
P *\left(Y_{i j}=t \mid \alpha_{i}\right)=\left(1-s_{j t}\right)^{\varphi_{i j t}} \cdot g_{j t}^{1-\varphi_{i j t}} \\
\varphi_{i j t}=\left\{\begin{array}{l}
1, \eta_{i j}^{*} \geq t \\
0, \text { otherwise }
\end{array}\right.
\end{gathered}
$$

In the above calculation process, formula (1), formula (5) and formula (7) are the project response probability function of PH-DINA model. Compared with P-DINA model, PH-DINA model not only increases the calculation of multi-level attribute indexes of subjects and projects, but also expands the calculation method of ideal response score, and increases the actual examination scope and feedback information of projects.

\section{Parameter estimation}

The parameter estimation of computer adaptive testing generally includes subject students' knowledge attribute condition estimation and project parameter condition estimation [16]. In order to give priority to ensuring the accuracy of project parameters, this paper improves the maximum likelihood estimation algorithm combined with the knowledge attribute parameters of PH-DINA model, 
but increases the time complexity to a certain extent. It still needs to be further optimized.

Assuming that $L\left(Y_{i} \mid \alpha\right)$ is the likelihood function of the subject $i$ under the multi-level attribute score, there are:

$$
\begin{aligned}
L\left(Y_{i} \mid \alpha\right)= & \prod_{j=1}^{J} \sum_{t=0}^{m f_{j}} P\left(Y_{i j}=t \mid \alpha\right)^{u_{i j t}} \\
u_{i j t} & =\left\{\begin{array}{l}
1, Y_{i j}=t \\
0, \text { otherwise }
\end{array}\right.
\end{aligned}
$$

Therefore, the likelihood function of PH-DINA model is:

$$
L\left(Y_{i} \mid \alpha\right)=\prod_{j=1}^{J} \sum_{t=0}^{m f_{j}}\left\lceil\left(1-s_{j t}\right)^{\varphi_{i j t}} \cdot g_{j t}^{1-\varphi_{i j t}}-\left(1-s_{j t+1}\right)^{\varphi_{i j t}} \cdot g_{j t+1}^{1-\varphi_{i j t+1}}\right\rceil^{u_{i j t}}
$$

Where, $u_{i j t}=\{0,1\}$ refers to the fact that the subject $i$ scored $t$ on item $j$, and the calculation formula of maximum likelihood estimation of the subject $i$ 's knowledge attribute is:

$$
\hat{a}_{i}=\arg \max \left\{L\left(Y_{i} \mid a_{c}\right)\right\}
$$

For $S$ and $g$ in the estimation of project parameters, accuracy is the primary factor to be considered, and the accuracy of project parameters will directly affect the accuracy of subjects' knowledge attributes. Therefore, MCMC (Markov Chain \& Monte Carlo) algorithm is selected to estimate them. MCMC algorithms are often used to solve high-dimensional integration and optimization problems, which are also the foundation of machine learning, physics, statistics, metrology, and other fields. The calculation accuracy of the algorithm is determined by the uniformity of sampling, which greatly simplifies the complexity of the problem. The hierarchical attribute structure is introduced into the project attribute parameters, and the prior probability distribution of the model

is $g_{j} \sim 4-\operatorname{Beta}(0,0.6,1,2), 1-s_{j} \sim 4-\operatorname{Beta}(0.4,1,2,1), \alpha_{i o} \sim U\left(0, L_{o}\right)$

According to Beyes theorem, the approximate conditional probability distribution of the parameters to be estimated is:

$$
P(s, g \mid Y, \alpha) \propto L(s, g \mid \alpha) P(s) P(g)
$$

Therefore, $\left\{s_{j}^{n+1}\right\}$ is randomly selected from uniform

distribution $U\left(s_{j}^{n}-\delta_{s}, s_{j}^{n}+\delta_{s}\right)$ and $\left\{g_{j}^{n+1}\right\}$ is randomly selected from uniform distribution $U\left(g_{j}^{n}-\delta_{g}, g_{j}^{n}+\delta_{g}\right)$. Now assuming $\delta_{s}=\delta_{g}=0.1$, the parameter transition probability formula is:

$$
p\left(\left\{s_{j}^{n}, g_{j}^{n}\right\},\left\{s_{j}^{n+1}, g_{j}^{n+1}\right\}\right)=\min \left\{\frac{L\left(s_{j}^{n+1}, g_{j}^{n+1} \mid \alpha_{i k}^{n+1}\right) P\left(s_{j}^{n+1}\right) P\left(g_{j}^{n+1}\right)}{L\left(s_{j}^{n}, g_{j}^{n} \mid \alpha_{i k}^{n+1}\right) P\left(s_{j}^{n}\right) P\left(g_{j}^{n}\right)}\right\}
$$

Since only the project's $j$ parameters of PH-DINA model need to be estimated, assuming that $N$ is the total number of people participating in the test, the effective likelihood function is:

$$
L(s, g \mid \alpha)=\left[\left\{\left(1-s_{j}\right)^{\eta^{\prime}{ }^{\prime j}} g_{j}^{1-\eta_{i j}^{\prime}}\right\}^{2} \cdot\left\{1-\left(1-s_{j}\right)^{\eta_{i j}^{\prime}} g_{j}^{1-\eta_{i j}^{\prime}}\right\}\right]
$$

\subsection{Personalized recommendation method of college P.E. teaching resources based on cognitive diagnosis model}

Aiming at the shortcomings of the personalized resource recommendation method based on collaborative filtering and the personalized resource recommendation method based on cognitive diagnosis [17], this paper proposes a new personalized recommendation method, the personalized recommendation method based on probability matrix decomposition and cognitive diagnosis model (PMF-CD method). This method combines the probability matrix decomposition method in collaborative filtering and PH-DINA model in cognitive diagnosis, so that the final personalized recommendation resources have good interpretability obtained by cognitive diagnosis and good recommendation effect obtained by probability matrix decomposition.

\section{Overall framework of PMF-CD method}

The PMF-CD method proposed in this paper is divided into three steps. The specific implementation process is as follows:

(1) Student cognitive diagnosis: PH-DINA model is used to model the mastery degree of students' knowledge points, obtain the mastery degree of students' knowledge points, and model the mastery level of students' resources according to the mastery degree of students' knowledge points.

(2) Student score prediction: combined with PMF method, the resource mastery of students after cognitive 
diagnosis and the examination of knowledge points to be recommended are introduced as a priori of student potential factors and project potential factors, so as to predict student project scores.

(3) Output: according to the actual needs, the project difficulty range of the resources is set to be recommended relative to the students to be recommended as $\left[\beta_{1}, \beta_{2}\right]$, and screen the resources whose probability of students completing the project correctly is within the recommended difficulty range, so as to make personalized resource recommendation to each student.

\section{Students' cognitive diagnosis process}

Because there are certain differences between the mastery degree of students' knowledge points and the difficulty of resources [18], only the information of students and students' resource selection is used for probability matrix decomposition to obtain the low-dimensional characteristics of students and resources, and predict that students' performance will lose some information. The lost information includes the difference in the mastery of knowledge points of different students and the difficulty of different resources [19]. Therefore, this paper uses PHDINA model to model the mastery degree of students' knowledge points.

When modelling, considering that the PH-DINA model may have shortcomings in giving the discrete mastery degree of students' knowledge points, this paper obtains the mastery degree of the probabilistic students' knowledge points by considering all possible $\alpha$ posterior probabilities, so that it can probabilistic model the mastery degree of students' knowledge points, simulate the mastery degree of students' knowledge points as a continuous value between 0 and 1 , redefine the estimated skill vector $\tilde{\alpha}_{i}$ and calculate student $Z_{i}$ 's mastery of skill $S_{k}$ according to the following formula (16):

$$
\tilde{\alpha}_{i k}=\frac{Z\left(\alpha_{x} \mid R_{i}\right) S}{r_{i v} \sum_{x=1}^{k} J Z\left(\alpha_{x} \mid R_{i}\right)}
$$

In the above formula, $R$ represents the score matrix of student resource; $Z$ represents the student set; $J$ is the resource set; ${ }^{r i v}$ is the student $i$ 's score on resource $v$; $S$ is the set of knowledge points; $k$ represents knowledge points; In the case of knowing the dispersion degree of students' knowledge points, this paper takes the observed students' resource response and corresponding resource skill needs as a priori to calculate the actual performance of students in resources, that is, the real level of students after excluding mistakes and guesses in the known students' response to resources.
For the true level $A_{i v}$ of student $Z_{i}$ 's response to resource ${ }^{J}$, this paper first uses geometric average to calculate the average skill mastery $S_{\text {avgiv }}$ of the student in the skills required by resources:

$$
S_{\text {avgiv }}=\sqrt[2]{\prod_{k=1}^{K} A_{i v} Z_{i} n_{k}}
$$

The value range of $n_{i k}$ is shown in formula (18):

$$
n_{i k}=\left\{\begin{array}{l}
1, q_{v k}=0 \\
\alpha_{i k} \cdot q_{v k}, q_{v k}=1
\end{array}\right.
$$

In the formula, $q_{v k}$ indicates whether the student has mastered the skill, 0 is the unmastered skill, and 1 is the mastered skill. When the average mastery of the skills required by the resources is known, taking the student's actual answer and the resource guess rate $g_{v}$ and error rate $S_{v}$ obtained by PH-DINA model as a priori parameters, the student's real level of resource learning can be calculated by using formula (19):

$$
A_{i v}=\left\{\begin{array}{l}
\frac{\left(1-s_{v}\right) S_{\text {avgiv }}}{\left(1-S_{v}\right) S_{\text {avgiv }}+g_{v}\left(1-S_{\text {avgiv }}\right)}, R_{i v}=1 \\
\frac{s_{v} S_{\text {avgiv }}}{S_{v} S_{\text {avgiv }}+\left(1-g_{v}\right)\left(1-S_{\text {avgiv }}\right)}, R_{i v}=0
\end{array}\right.
$$

Thus, the first step of cognitive diagnosis is completed.

\section{Student score prediction process}

After the student's cognitive diagnosis information is obtained, it is used for probability matrix decomposition. In fact, feature $b_{i v}$ can be extracted from the student's actual level matrix $A$ as the prior information of PMF:

$$
\begin{aligned}
& b_{i v}=b_{i}+b_{v} \\
& b_{i}=\frac{1}{V} \times \sum_{o=1}^{U} A_{i o} \\
& b_{v}=\frac{1}{U} \times \sum_{o=1}^{U} A_{o v}
\end{aligned}
$$


Where, ${ }^{b}{ }$ represents the prior degree of learning score of student $Z_{o}$, which reflects the difference in the mastery of knowledge points among students, and is the average value of line $i$ of matrix $A ;{ }^{b}$ represents the prior score of resource $v$, which reflects the difference in difficulty between different resources. It is the average value of column $v$ of matrix $A$. By considering the mastery of students' knowledge points, $b_{i}$ and $b_{v}$ can truly reflect students' personality learning status.

When the prior functions $b_{i}$ and $b_{v}$ of students and resources are added, the modelling of response based on cognitive diagnosis is completed. In this model, the potential response $\eta_{i v}$ of students is calculated by the following formula (21):

$$
\eta_{i v}=\mu+\rho b_{i v}+(1-\rho) M_{i} N_{v}
$$

Where, ${ }^{\mu}$ is the overall average score. In this formula, the proportion of students' individual learning state and common learning state among students in $\eta_{i v}$ prediction is adjusted by parameter $\rho, \rho \in[0,1]$. When $\rho$ is greater, it means that the prediction score is more affected by the common learning state of students. When $\rho=0$, students' individual learning state is not introduced, and this method degenerates into PMF method. By adding students' personalized learning state $b_{i v}$ to the PMF decomposition, the PMF can decompose the lowdimensional potential factor $M, \mathrm{~N}$, and students' personality characteristics are added when including the common learning state between students. Therefore, the accuracy of score prediction and the interpretability of results can be improved.

In the process of student $\mathrm{Q} \& \mathrm{~A}$ prediction in this paper, it is composed of four parts: overall average value, resource a priori, student a priori and student resource. Each part explains a certain attribute of the observed value, so the optimization objective of PMF-CD can be changed into the minimization function formula:

$$
E=\sum_{i=1}^{U} \sum_{v=1}^{V} I_{U V}\left(R_{i v}-\eta_{i v}\right)^{2}+\lambda_{M}\|M\|_{F r o}^{2}+\lambda_{N}\|N\|_{F r o}^{2}
$$

In the above formula, $\lambda_{M}$ and $\lambda_{N}$ are the regularization coefficients of the model; $\eta_{i v}$ represents the student $i$ 's ideal response score to resource $v$.
In general, when using PMF-CD method in the modelling of students' resource response, by combining the interpretable information such as students' knowledge points and resource difficulty, it makes the method interpretable with the cognitive diagnosis model, and eliminates the interference of students' guesses and mistakes on the modelling of resource response to the greatest extent.

\section{Personalized recommendation of teaching resources based on student score prediction}

After obtaining the students' potential answers $\eta_{i v}$, the last step of the PMF-CD method is to recommend personalized resources to different students according to the students' potential answers. In this part, this paper describes the PMF-CD method to obtain personalized resource recommendations according to the students' potential answers.

In the context of personalized resource recommendation, different from other interest point recommendation methods, it is not the test questions, the most difficult resources or the simplest resources that students are most interested in [20]. Therefore, in the process of PMF-CD resource recommendation, the difficulty of recommended resources can be set according to students' needs. Resource difficulty refers to the difficulty of items in resources to students. The probability that student $Z_{i}$ correctly completes resource project $J_{v}$ is taken as the difficulty $D_{i v}$ of resource $J_{v}$ for student $Z_{i}$, that is:

$$
D_{i v}=Z\left(r_{i v}=1 \mid Z_{i}, J_{v}\right)
$$

When recommending resources, the difficulty ranges $\beta_{1}$ and $\beta_{2}\left(\beta_{1}<\beta_{2}\right)$ of recommended resources are set as the boundary of the difficulty of recommended resources. PMF-CD method can recommend to student $Z_{i}$ the resource set $J_{\text {rec }}$ with the correct response probability between $\beta_{1}$ and $\beta_{2}$ from the resource set $J_{r e c_{-} u}$ to be recommended according to the students' potential response $\eta_{i v}$ :

$$
J_{r e c_{-} i}=\left\{J_{v} \mid J_{v} \in J_{r e c}, D_{i v} \in\left[\beta_{1}, \beta_{2}\right]\right\}
$$

Where $\beta_{1}$ is the lower bound of students' correct completion probability and $\beta_{2}$ is the upper bound of students' correct completion probability. Therefore, after setting the corresponding difficulty range of 
recommended test questions, $\mathrm{PMF-CD}$ can recommend resources within the corresponding difficulty range for each student.

Because PMF-CD method effectively combines the individuality of students' own learning situation and the commonness of learning situation among students in resource recommendation, the method in this paper can effectively recommend personalized resources suitable for difficulty to students.

\section{Experimental analysis}

In this paper, a total of 28,374 pieces of answering data collected by the laboratory and the smart classroom online teaching cloud platform of a certain university were collected and named as the DATA data set. This paper extracts $70 \%$ of the dataset as the test dataset and the rest as the training dataset. The experimental data is automatically exported by the teaching cloud platform. The experiment runs on Windows 10 operating system, the CPU is Intel i7-4790, the graphics card is NVIDIA GTX 1070, and the memory is $32 \mathrm{G}$. This paper applies the proposed method to a learning platform of a university, makes personalized recommendation on the data set of physical education teaching resources in the university, and selects the methods in reference [8], reference [9] and reference [10] to compare with the proposed method.

The selection strategy of adaptive test is adopted, and the unconstrained evaluation index is used to verify the effect of the method recommended in this paper. The unconstrained index mainly includes resource security and testing efficiency (TE), in which security includes resource exposure ratio (ER) and testing overlap ratio (TOR).

ER reflects the exposure degree of the resource library, and generally adopts chi square calculation indicators:

$$
\chi^{2}=\sum_{j=1}^{M} \frac{\left[E R_{j}-E\left(E R_{j}\right)\right]^{2}}{E\left(E R_{j}\right)}
$$

Where $E R_{j}=f_{j} / N$ is the exposure rate of the $j_{\text {-th }}$ item, $f_{j}$ is the number of times the $j$-th item is extracted, $E\left(E R_{j}\right)$ is the expected exposure rate of the $j_{\text {-th item, and the smaller the value of }} E R_{j}$ is, the lower the exposure rate and the higher the safety are. The ideal situation in the test process is that all test questions can be evenly extracted, that is, $E\left(E R_{j}\right)=\Upsilon / \Psi, \Upsilon$ is the average test length, and $\Psi$ is the total number of items in the resource database. Therefore, $\chi^{2}$ is used to count the distance between the observed exposure rate and the expected exposure rate. The smaller the $\chi^{2}$ value is, the higher the security of resources is.

TOR reflects the overlap of the same resources extracted by different subjects. Therefore, its calculation formula is directly related to the topic exposure rate, test length and the number of subjects. The higher the overlap rate is, the lower the safety of the test items is. The calculation formula is as follows:

$$
\overline{\hat{T}}=\frac{N \times \sum_{j=1}^{\Psi} E R_{j}^{2}}{(N-1) \times \Upsilon}-\frac{1}{N-1}
$$

TE is an index for comprehensive evaluation of test efficiency ratio, which refers to the average amount of resources consumed under the same measurement accuracy. The lower the TE value is, the higher the efficiency is. Where, $\Upsilon_{i}$ refers to the number of items the students $i$ 's average consumed during the test.

$$
T E=\sum_{i=1}^{N} \Upsilon_{i} / N
$$

The changes of various indicators of different recommended methods are analyzed during recommendation, and the fixed measurement accuracy is set as $0.6,0.7,0.8$ and 0.9 respectively. Under the fixed measurement accuracy, the recommendation effect analysis of each method is shown in Table 1.

According to Table 1, the proposed method is obviously superior to other methods in all aspects. Regarding the experiments of ER indicators, the ER values of the three reference methods are all higher than 20, while the ER value measured by the reference [10] method is up to 62.21, which is less safe. The ER values of the method in this paper are all lower than 1 , and its safety is much higher than that of the methods in the three references. Regarding the experiment of the TOR indicator, the TOR value of the method in this paper is always lower than 0.01 , indicating that the overlap rate of students extracting the same resources is low, and the recommendation effect is more accurate. The TOR value measured by the method in Reference [8] is also significantly higher than other methods, which cannot achieve accurate cognitive diagnosis of students, resulting in an inaccurate recommendation effect. Regarding the experiment of TE index, the method in this paper can recommend teaching resources to students with less resource consumption. To sum up, the method in this paper can effectively guarantee the resource security. This is because the method in this paper can construct a model of students' resource mastery level according to the relationship between students' existing resource responses 
and resource knowledge points, thereby improving the

efficiency of personalized recommendation.

Table 1. Comparison of test results of recommended methods

\begin{tabular}{|c|c|c|c|c|}
\hline Different methods for & Accuracy of measurement & ER & TOR & $\mathrm{TE}$ \\
\hline \multirow{4}{*}{ In this paper, methods } & 0.6 & 0.16 & 0.06 & 3.47 \\
\hline & 0.7 & 0.17 & 0.07 & 4.35 \\
\hline & 0.8 & 0.19 & 0.07 & 5.54 \\
\hline & 0.9 & 0.21 & 0.08 & 6.32 \\
\hline \multirow{4}{*}{ Methods of reference [8] } & 0.6 & 42.33 & 0.34 & 5.86 \\
\hline & 0.7 & 44.64 & 0.36 & 6.65 \\
\hline & 0.8 & 47.53 & 0.37 & 6.97 \\
\hline & 0.9 & 49.53 & 0.39 & 7.53 \\
\hline \multirow{4}{*}{ Methods of reference [9] } & 0.6 & 29.35 & 0.23 & 10.64 \\
\hline & 0.7 & 32.63 & 0.25 & 10.86 \\
\hline & 0.8 & 35.64 & 0.27 & 11.64 \\
\hline & 0.9 & 38.47 & 0.28 & 12.64 \\
\hline \multirow{4}{*}{ Methods of reference [10] } & 0.6 & 56.32 & 0.31 & 13.53 \\
\hline & 0.7 & 58.53 & 0.32 & 14.64 \\
\hline & 0.8 & 59.21 & 0.34 & 14.98 \\
\hline & 0.9 & 62.21 & 0.34 & 15.67 \\
\hline \multicolumn{3}{|c|}{$\begin{array}{l}\text { Among them, the ER value measured by the method in } \\
\text { reference [10] is always the highest, indicating that this } \\
\text { method has a high resource exposure rate and is not } \\
\text { suitable for resource personalized recommendation. } \\
\text { Although the resource exposure rate of the other two } \\
\text { methods is lower than that of the method in this paper, it } \\
\text { is still much higher than that of the method; in this paper } \\
\text { The TOR value measured by the method in reference [8] } \\
\text { is also significantly higher than that of other methods. } \\
\text { Therefore, it can be seen that this method has a high test } \\
\text { overlap rate and can not achieve accurate student }\end{array}$} & \multicolumn{2}{|c|}{$\begin{array}{l}\text { cognitive diagnosis, resulting in inaccurate } \\
\text { recommendation effect. When analyzing the security and } \\
\text { test efficiency of resources, the method in this paper is } \\
\text { always the lowest under different measurement accuracy, } \\
\text { which shows that this method can effectively ensure the } \\
\text { security of resources and improve the efficiency of } \\
\text { personalized recommendation. } \\
\text { The recommendation ability of different methods is } \\
\text { analyzed from the aspects of application, real-time and } \\
\text { cold start ability of personalized recommendation. The } \\
\text { analysis results are shown in Table } 2 \text {. }\end{array}$} \\
\hline
\end{tabular}

Table 2. Analysis of recommendation ability of different methods

\begin{tabular}{|c|c|c|c|c|}
\hline Performance & The proposed method & Method in Ref.8 & Method in Ref.9 & Method in Ref.10 \\
\hline Applied & $\begin{array}{l}\text { It focuses on students' own cognitive } \\
\text { diagnosis, which is suitable for the } \\
\text { situation where students' individual } \\
\text { needs are not obvious. } \\
\text { Recommended resources are easy to } \\
\text { explain and describe, and have } \\
\text { higher recognition and acceptance. }\end{array}$ & $\begin{array}{l}\text { This method is difficult } \\
\text { to find the content that } \\
\text { users ignore or potential } \\
\text { interest, and requires a } \\
\text { large amount of data as } \\
\text { support. }\end{array}$ & $\begin{array}{l}\text { This method also needs the } \\
\text { support of large amount of } \\
\text { data as the basis, so the } \\
\text { calculation is very large and } \\
\text { time-consuming in the } \\
\text { application process. }\end{array}$ & $\begin{array}{l}\text { Poor scalability, } \\
\text { once the method is } \\
\text { applied or } \\
\text { established, it is } \\
\text { difficult to modify or } \\
\text { make flexible } \\
\text { changes. }\end{array}$ \\
\hline Real time & $\begin{array}{l}\text { When making recommendations, the } \\
\text { recommendation list is more accurate } \\
\text { and stable, and it will change in real } \\
\text { time when users browse items and } \\
\text { have changes. }\end{array}$ & $\begin{array}{l}\text { The recommendation list } \\
\text { cannot be changed in } \\
\text { real time and requires } \\
\text { the user to enter more } \\
\text { association information. }\end{array}$ & $\begin{array}{l}\text { Real-time performance is } \\
\text { weak, and repeated content } \\
\text { recommendation may occur } \\
\text { after a recommendation is } \\
\text { completed. }\end{array}$ & $\begin{array}{l}\text { Data is static and } \\
\text { cannot be } \\
\text { dynamically changed } \\
\text { in real time. }\end{array}$ \\
\hline Cold start & $\begin{array}{l}\text { When users browse the project, the } \\
\text { recommendation platform will } \\
\text { calculate and generate the } \\
\text { recommendation list according to the } \\
\text { current project, which will not cause } \\
\text { cold start problems for new users. }\end{array}$ & $\begin{array}{l}\text { The cold start problem } \\
\text { cannot be completely } \\
\text { avoided, and it takes a } \\
\text { lot of time to calculate } \\
\text { the similarity between } \\
\text { projects. }\end{array}$ & $\begin{array}{l}\text { High requirements on data } \\
\text { structure, so the application } \\
\text { range is poor, cold start } \\
\text { problem is inevitable. }\end{array}$ & $\begin{array}{l}\text { There are data cold } \\
\text { start and data } \\
\text { sparsity problems. }\end{array}$ \\
\hline
\end{tabular}


According to Table 2, the applicability of the three comparison methods is poor, and the method in reference [10] does not have high scalability. At the same time, the method in this paper has high resource recognition and acceptance when recommending, so it has good applicability. After practical application, the method in this paper has good real-time performance, and the recommendation list can be changed in real time according to the browsing records of students. This method will not have the problem of cold start, which is inevitable for other methods, and the three comparison methods are time-consuming.

Mean absolute error (MAE) and F1 index are used to evaluate whether the method in this paper can effectively recommend personalized resources to students.

F1 value: it is the easiest way to measure the difference between the real value and the predicted value. The recommended effect is verified by predicting whether the students have completed the answer and the deviation of the students' actual answer. The calculation formula is shown in (28):

$$
F 1=\frac{2 \times \text { precison } \times \text { recall }}{\text { precison }+ \text { recall }}
$$

Mean absolute error (MAE): it is a commonly used index to evaluate the performance of recommendation methods. It is the calculation of the average absolute error between the predicted value and the real value of learners in the process of answering resources. The formula is shown in (29):

$$
M A E=\frac{\sum_{i=1}^{N}\left|\kappa_{i}-\imath_{i}\right|}{\vartheta}
$$

Where, $\vartheta$ is the number of resources, $\kappa_{i}$ is the predicted score, and $l_{i}$ is the actual score. When the MAE value is larger, the recommendation effect is worse, and the MAE value is smaller, the recommendation effect is better.

The experimental data are randomly divided into test training sets according to different proportions. Experiments are carried out on test sets with different proportions by using different recommendation methods. The mean absolute error (MAE) and F1 value under each method are calculated, and the recommendation effects of three different recommendation methods are compared, analyzed and evaluated. The analysis results are shown in Figure 2.

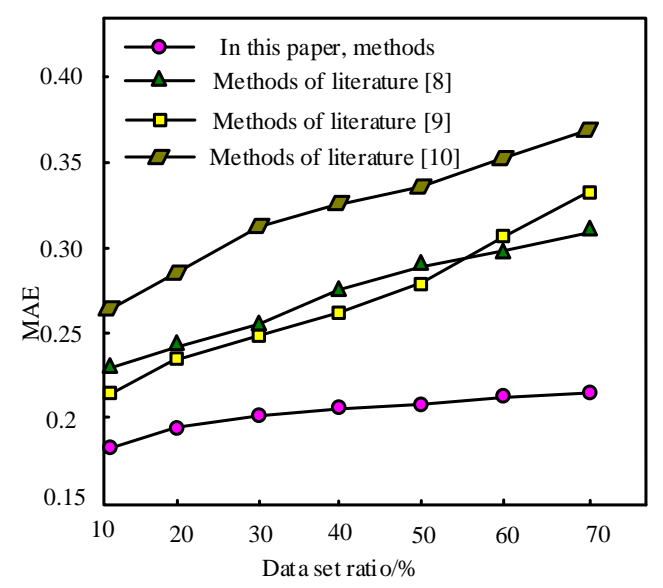

(a) Mean absolute error analysis

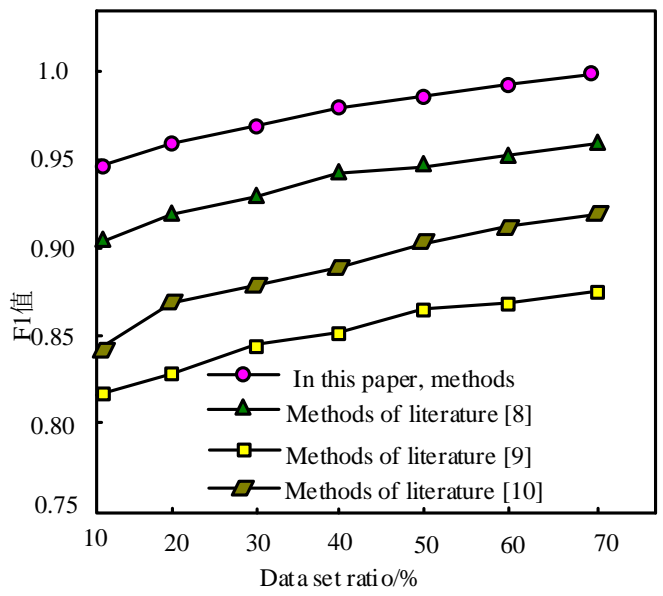

(b) Analysis of F1 value

Figure 2. Performance analysis of different recommended methods

According to Figure 2, it can be seen that the recommendation error of the method in Reference [10] is the highest when the proportion of the dataset is not passed, and the highest value is 0.38 , indicating that this method cannot accurately implement resource recommendation. The MAE values of the reference [8] method and the reference [9] method are similar, both higher than 0.22. However, the MAE values of the methods in this paper are all less than 0.22 , indicating that the recommendation results of teaching resources are more accurate. The F1 value of the method in the literature [9] is the lowest, indicating that the recommended results do not match the actual situation. However, the method in this paper has the lowest MAE and the highest F1 value, which is 0.98 , which indicates that the method in this paper has a lower error in the recommendation and the result is more accurate. Because the method in this paper is used to decompose the knowledge mastery attribute model of the students tested by the probability matrix, it can accurately predict the students' answering situation, so as to recommend the 
corresponding resources to the students according to the students' score prediction and resource difficulty.

\section{Discussion}

In view of the defects of traditional cognitive diagnosis model and traditional PMF algorithm in student resource recommendation, that is, the former ignores the learning personality among students and the latter ignores the learning personality of student groups, this paper improves the cognitive diagnosis model PH-DINA with multi-level multi-attribute score, and gives the PHHKL topic selection strategy and parameter estimation method used in the same mode. It makes up for the deficiency of the existing adaptive test in dealing with multi-level attribute scoring data. At the same time, the personalized resource recommendation method PMF-CD is realized by combining probability matrix decomposition and cognitive diagnosis model. In this method, firstly, the mastery degree of students' knowledge points is modeled in combination with the cognitive diagnosis model, and the mastery degree of students' resources is obtained by investigating the knowledge points of resources. Then, the mastery degree of students' personalized resources is used as a priori information of PMF algorithm to predict students' scores. Considering the commonness of learning among the student groups, the individual learning personality status of the recommended students is added. Finally, according to the difficulty range of the recommended resources, the resources suitable for the students themselves are selected to form a set of resources to be recommended and recommended to the students. Experiments show that the recommendation method comprehensively considers the individual characteristics and the common characteristics of the group, and improves the interpretability and reliability.

This is the body text with indent. This is the body text with indent. This is the body text with indent. This is the body text with indent. This is the body text with indent. This is the body text with indent. This is the body text with indent.

\section{Conclusion}

(1) The continuous extension and sharing of teaching resources is accompanied by the problem of data "explosion", even if the use of a search engine in the system can help students quickly find the teaching resources they need to find. However, in many cases, students do not know exactly what they need. Or students can't express what they need. Therefore, it is very useful to provide physical education teaching recommendations for students according to their different needs and their own hobbies.

(2) The personalized recommendation method of college P.E. teaching resources based on cognitive diagnosis model is based on cognitive diagnosis model, and carries out personalized recommendation for different physical education teaching resources.

(3) This method makes up for the lack of diagnosis and interpretation of push content in other push methods. In addition, this method diagnoses at the cognitive level and integrates students' own learning characteristics, so the push content is more accurate and effective.

(4) In future research, we will consider applying this method to mobile learning to further protect the privacy performance of user data.

\section{References}

[1] Park, S., Yi, H. S., \& Han, S. K. (2019). An exploratory study on the analysis of the digital literacy test based on the cognitive diagnostic theory. Korean Association for Educational Information and Media, 25(2), 373-402.

[2] Wang, S., Liu, X., Liu, S., et al. (2022). Human ShortLong Term Cognitive Memory Mechanism for Visual Monitoring in IoT-Assisted Smart Cities. IEEE Internet of Things Journal, online first, 10.1109/JIOT.2021.3077600

[3] Liu, S., He, T., Dai, J. (2021). A Survey of CRF Algorithm Based Knowledge Extraction of Elementary Mathematics in Chinese. Mobile Networks \& Applications, 26(5), 18911903

[4] Li, H., Zhang, S., Shi, J., \& Hu, Y. (2019). Research and design of intelligent learning system based on recommendation technology. Control \& Intelligent Systems, 47(1), 43-49.

[5] Saito, T., Watanobe, Y. (2020).Learning path recommendation system for programming education based on neural networks. International Journal of Distance Education Technologies, 18(1), 36-64.

[6] Yuan, Q. (2020). Network education recommendation and teaching resource sharing based on improved neural network. Journal of Intelligent and Fuzzy Systems, 39(4), 5511-5520.

[7] Lin, X., Zhang, M., Liu, Y., \& Ma, S. (2019). Enhancing personalized recommendation by implicit preference communities modeling. ACM Transactions on Information Systems, 37(4), 1-32.

[8] Wei, J., Meng, F. (2019).Personalized information recommendation based on synonymy tag optimization. Cluster Computing, 22(3), 5467-5478.

[9] Wu, W., \& Yin, B. (2019).Personalized recommendation algorithm based on consumer psychology of local group purchase e-commerce users. Journal of Intelligent and Fuzzy Systems, 37(5), 5973-5981.

[10] Zhou, W., \& Han, W. (2019).Personalized recommendation via user preference matching. Information Processing \& Management, 55(3), 955-968.

[11] Akbay, L., Torre, J. (2020). Estimation approaches in cognitive diagnosis modeling when attributes are hierarchically structured. Psicothema, 32(1), 122-129.

[12] Ozaki, K., Sugawara, S., \& Arai, N. (2020).Cognitive diagnosis models for estimation of misconceptions analyzing multiple-choice data. Behaviormetrika, 47(1), $19-41$.

[13] Liu, S., Wang, S., Liu, X., et al. (2021). Human Memory Update Strategy: A Multi-Layer Template Update Mechanism for Remote Visual Monitoring, IEEE Transactions on Multimedia, 23, 2188-2198 
[14] RQMD, C, Pompeu, J. E., Viveiro, L., \& SMD B. (2020). Spatial orientation tasks show moderate to high accuracy for the diagnosis of mild cognitive impairment: a systematic reference review. Arquivos de NeuroPsiquiatria, 78(11), 713-723.

[15] Gündüz, T Akan, M. (2020).The classification of students based on mathematics achievement test in timss 2015 turkey sample by cognitive diagnosis models. Necatibey Eğitim Fakültesi Elektronik Fen ve Matematik Eğitimi Dergisi, 14(2), 1040-1079.

[16] Duan, H., \& Jiang, Y. (2020).Whether examinees' reading abilities are in line with examination structure - from the perspective of cognitive diagnosis. Linguistics, 2(3), 151164.

[17] Shuai, L., Dongye, L., Srivastava, G., et al. (2021). Overview and methods of correlation filter algorithms in object tracking. Complex \& Intelligent Systems, 7, 18951917

[18] Choi, I.Y., Moon, H.S., \& Kim, J.K. (2019). Assessing personalized recommendation services using expectancy disconfirmation theory. Asia Pacific Journal of Information Systems, 29(2), 203-216.

[19] Zhou, L., Zhang, F., Zhang, S., \& Xu, M. (2021). Study on the personalized learning model of learner-learning resource matching. International Journal of Information and Education Technology, 11(3), 143-147.

[20] Gao, P., Li, J., Liu, S. (2021). An Introduction to Key Technology in Artificial Intelligence and big Data Driven e-Learning and e-Education. Mobile Networks \& Applications, 26(5), 2123-2126 